Desarrollo de capacidades de investigación para estudiantes

\author{
universitarios mediante el uso
}

de estrategias instruccionales en
Developing research capacities

for undergraduate students using

instructional strategies in virtual

learning environments

\title{
entornos virtuales de aprendizaje
}

\author{
Danilo Pástor Ramírez* | Gloria de Lourdes Arcos Medina** | Agustín Lagunes Domínguez***
}

Recepción del artículo: 1/10/2019 | Aceptación para publicación: 11/2/2020 | Publicación: 30/3/2020

\section{RESUMEN}

Este artículo contribuye al desarrollo de capacidades de investigación en los estudiantes universitarios mediante el uso estrategias instruccionales en entornos virtuales de aprendizaje; esto, con la finalidad de que los estudiantes obtengan las habilidades y destrezas necesarias, y que puedan mejorar sus procesos de investigación. Para determinar cuáles son las capacidades de investigación a desarrollar, se realiza una revisión de literatura con el objetivo de identificar los estudios afines al objeto abordado. También se examinan las estrategias instruccionales vinculadas a las capacidades de investigación de los estudiantes de pregrado analizadas. Se describe el aula virtual diseñada y aplicada a los estudiantes para que adquieran las capacidades propuestas, para validar estas, se hace una evaluación a los estudiantes a fin de establecer el nivel de desarrollo adquirido en cuanto a las capacidades mencionadas, a través de un cuestionario que mide sus conocimientos, el cual arrojó una mejora global en la aplicación de las competencias investigativas del $13.5 \%$, lo que demuestra la validez del uso de las estrategias instruccionales en un entorno virtual.

\section{Abstract}

This paper contributes to the development of research skills in university students, through the use of instructional strategies in virtual learning environments, in order to achieve that students obtain the necessary skills and abilities to improve their research processes. To determine which are the research capacities to be developed, a literature review is carried out, whose objective is to identify the studies that are suitable with the teaching strategies. An analysis of the instructional strategies that can be carried out with the research capabilities of undergraduate students is also performed. The virtual classroom designed to be applied to students in teaching research skills is described. To validate the proposed competencies, the students were evaluated to determine the level of development of the research competencies through a questionnaire that measures their knowledge and a global improvement in the application of the investigative competences of $13.5 \%$ is obtained, demonstrating the validity of the use of instructional strategies through a virtual environment.

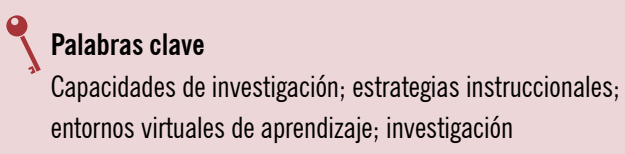

\section{Keywords \\ Research capacities; instructional strategies; virtual learning environment; research}

* Doctor en Ingeniería - Sistemas e Informática por la Universidad Nacional de Colombia. Profesor investigador de la Escuela Superior Politécnica de Chimbora- 


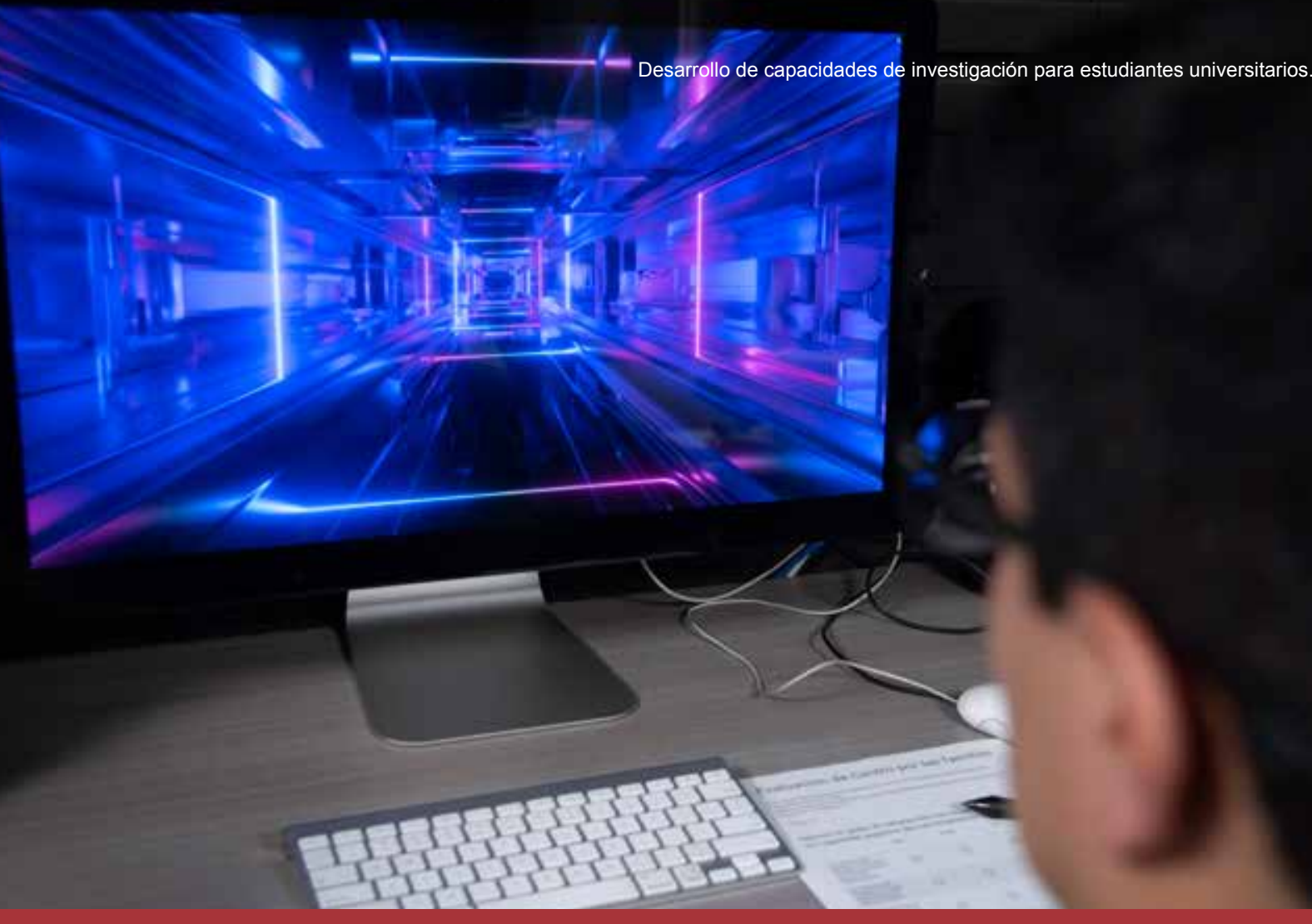

\section{INTRODUCCIÓN}

$\mathrm{E}$ n la actualidad, la educación superior se centra en la formación de destrezas y habilidades en los estudiantes universitarios para promover su preparación integral. Para adquirir capacidades pertinentes a la investigación, una alternativa es el diseño de un plan de instrucción complementario a través de estrategias de enseñanza innovadoras y alternativas que permitan desarrollar estas capacidades. Esto implica que, para crear la habilidad de producir y utilizar nuevos conocimientos, se debe fortalecer la cultura de investigación en la universidad, que constituye un elemento fundamental en el proceso educativo y aporta a la generación de nuevo conocimiento (González, 2004). Por esta razón, las universidades deben fomentar la obtención de capacidades de investigación en los estudiantes e incorporarlas como una estrategia de enseñanza-aprendizaje en el currículo (Miyahira Arakaki, 2009).
En el ámbito de educación superior, las competencias investigativas son capacidades que los docentes deben poseer para comprender el proceso de investigación y cumplir a cabalidad su papel de impartidor de conocimientos (Lau et al., 2015); aunque los estudiantes de pregrado apenas se forman con habilidades y destrezas de investigación básicas, que aprenden como experiencia práctica, sin ser parte de su malla curricular, e indistintamente de su tipo de carrera. Por el contrario, si se establecieran bases para que un formador practique la investigación, entonces, por sí mismo, aportaría al desarrollo de un espíritu investigativo en sus estudiantes y tendría la capacidad de originar experiencias de enseñanza por descubrimiento y construcción del conocimiento, centradas en el potencial de cada estudiante (García-Gutiérrez y Aznar-Díaz, 2018).

El desarrollo de la capacidad de investigación se define como un proceso de desarrollo individual que conduce a niveles más altos de habilidad para 
realizar investigaciones útiles (Moore, Crozier \& Kite, 2012; Trostle, 1992). En general, las universidades exigen tomar cursos para adquirir habilidades investigativas en los niveles de posgrado, pero obtener estas capacidades en entornos académicos de pregrado no es responsabilidad del programa de formación. El principal problema asociado con los estudiantes de pregrado es hacer coincidir el nivel de conocimientos que posee el estudiante con sus capacidades de investigación.

Este problema se puede minimizar con la aplicación de estrategias de enseñanza especializadas en áreas específicas de investigación que el estudiante requiera (Beckerleg \& Collins, 2007); sin embargo, existen inconvenientes que apuntan a la necesidad de buscar indicios que ayuden a diseñar, estructurar y compartir de manera más eficiente un conjunto de estrategias didácticas, $\mathrm{y}$ que colaboren a la autoformación para la investigación. Estas estrategias ayudarían al estudiante de pregrado a enfocarse en propósitos relevantes, como la búsqueda, identificación y puesta en práctica de habilidades investigativas que aportan nuevos conocimientos (Reyes, 2013).

La formación y el desarrollo de las capacidades de investigación son una tendencia en la literatura científica. Entre los trabajos en este ámbito, podemos citar los de Barth et al. (2007); Beckerleg y Collins (2007); Davidson y Palermo (2015); Emelyanova, Teplyakova y Boltunova (2017); Hughes (2019); e Ismuratova et al. (2018), los cuales proponen competencias, habilidades y capacidades de investigación orientadas a un nivel de posgrado, especialización o doctorado. A pesar de que estos estudios han contribuido a un análisis para determinar los mejores precedentes en el campo de las capacidades de investigación, resultan insuficientes debido a que no se orientan hacia un enfoque pedagógico o sistemático apoyado en técnicas o estrategias instruccionales específicas. Tampoco se menciona en estos estudios que las capacidades de investigación se sustentan en el uso de entornos virtuales de aprendizaje (EVA).
El objetivo de este trabajo es desarrollar capacidades de investigación en los estudiantes universitarios mediante el uso de estrategias instruccionales apoyadas en los EVA para mejorar los procesos de investigación. Para este fin, evaluamos las capacidades de investigación de 54 estudiantes y aplicamos técnicas de enseñanza adecuadas con material de aprendizaje virtual; de esta manera, demostramos una mejora en la adquisición de las capacidades propuestas.

El documento está organizado de la siguiente manera: en un primer apartado, exponemos los trabajos relacionados, planteamos el problema y justificamos el objetivo de la investigación; en el segundo, describimos el procedimiento para determinar las capacidades de investigación consideradas en el estudio; posteriormente, especificamos la metodología usada para establecer las estrategias instruccionales acorde con el estudio planteado, crear el entorno virtual y evaluar las capacidades de investigación; finalmente, realizamos un análisis de los resultados de la evaluación de las capacidades propuestas y detallamos las conclusiones del estudio.

\section{DETERMINACIÓN DE CAPACIDADES DE INVESTIGACIÓN}

Actualmente, las universidades tienen una necesidad imperiosa de desarrollar las capacidades de investigación en los estudiantes para mejorar la calidad de la educación. A pesar de que en las instituciones educativas estas capacidades califican como un elemento transversal en las carreras de educación superior, es conveniente que sean consideradas como el resultado de aprendizajes genéricos para los graduados (Coates, 2014), de tal forma que estos obtengan un determinado nivel de estas capacidades a través de estrategias de enseñanza y propicien la construcción del conocimiento.

Para determinar cuáles son las capacidades de investigación que se debían implementar en un 
curso de formación de estudiantes universitarios, revisamos la literatura cuyo objetivo era identificar los estudios de las capacidades de investigación que tuvieran relación con estrategias de enseñanza. Realizamos una búsqueda avanzada en Scopus y en Google Scholar, y restringimos el rango desde 2005 hasta 2019. Los términos de búsqueda incluidos fueron los siguientes: research capacities, education, instructional strategies, e-learning, higher education y virtual course. También consideramos algunos sinónimos de los términos buscados en inglés. A partir de estos resultados, seleccionamos los estudios que promueven algún tipo de capacidades de investigación. En los siguientes párrafos, sintetizamos los trabajos que nos ayudaron a determinar las capacidades propuestas en esta investigación.

El estudio de Emelyanova et al. (2017)formula capacidades de investigación para el grado de máster; no obstante, señala que deben cumplirse cierto tipo de actividades que los estudiantes de pregrado deben aprender durante la capacitación universitaria, y las cuales incluyen el uso de métodos de investigación, formulación, análisis y resolución de problemas de investigación. En otro trabajo, Ismuratova et al. (2018) mencionan que la capacidad investigadora de los estudiantes universitarios se caracteriza por su participación en una serie de trabajos diferentes: reportes, do- cumentos de referencia, trabajos de investigación y proyectos, lo cual implica dominar los métodos modernos de búsqueda, procesamiento y uso de la información, así como algunos métodos de investigación científica.

Por su parte, Davidson y Palermo (2015) proponen la enseñanza de habilidades de investigación como parte de un desarrollo curricular en cursos de pregrado y plantean los siguientes resultados de aprendizaje: diseño de estudio de investigación, desarrollo de una revisión sistemática de literatura, gestión de proyectos de investigación, comparación de metodologías de investigación, aplicación de métodos estadísticos de análisis de datos cualitativos y cuantitativos, y comunicación de resultados de literatura científica. De la misma forma, Tobón (2013) describe las principales capacidades de investigación, las cuales se refieren a las habilidades lectoras y de escritura, la gestión de la información, el aprendizaje autónomo, el espíritu de búsqueda e indagación, así como hábitos de estudio sólidos.

En el mismo contexto, Lagunes, Judikis y Flores (2016) proponen capacidades concernientes a nueve conocimientos y 21 habilidades que un estudiante universitario debe poseer. Las relacionadas con las capacidades de investigación son: identificar problemas del proyecto de investigación, realizar análisis cualitativo y cuantitativo de

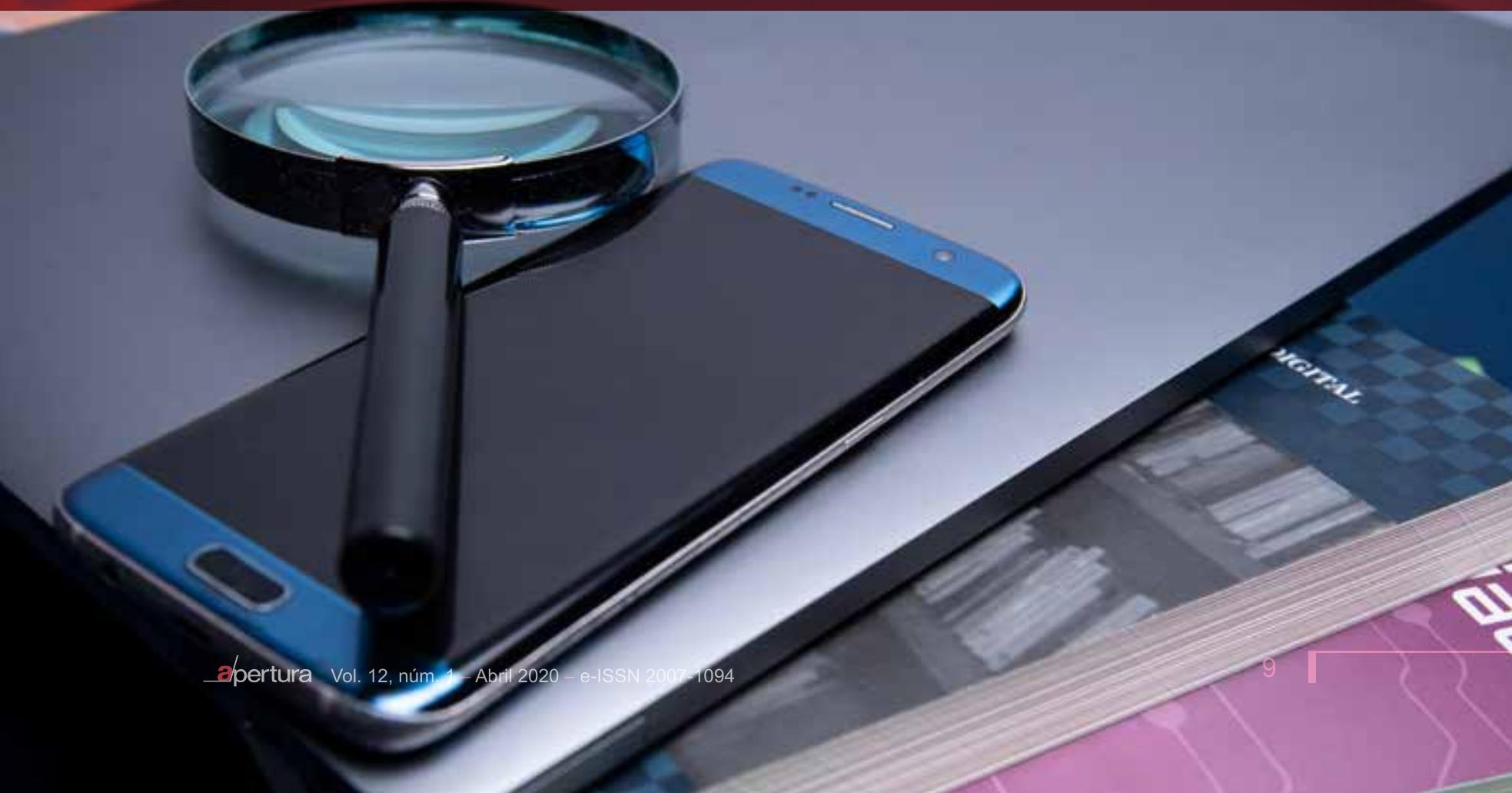


datos, hacer búsqueda de información relevante, analizar y resolver problemas; analizar, buscar y evaluar información, entre otras. En la literatura presentada por Rivas (2011) se precisan nueve capacidades del modelo LART 2011. Nosotros consideramos necesario restringir a un nivel universitario las habilidades para plantear el problema de la investigación, revisar el estado del arte, conocer técnicas de análisis de datos y redactar los resultados científicos. La tabla 1 resume la propuesta de estos autores para determinar las capacidades de investigación.

Después de analizar la literatura encontrada en la revisión bibliográfica, hemos seleccionado cinco capacidades de investigación que consideramos fundamentales para el proceso del aprendizaje en estudiantes universitarios, y que describimos a continuación.

Tabla 1. Resumen de los trabajos que proponen capacidades de investigación

\begin{tabular}{|c|c|c|}
\hline TíTULO DEL ESTUDIO & AUtOR(ES) & CAPACIDADES DE INVESTIGACIÓN \\
\hline $\begin{array}{l}\text { The students' research } \\
\text { competences formation } \\
\text { on the master's program- } \\
\text { mes in Pedagogy }\end{array}$ & Emelyanova et al. (2017) & $\begin{array}{l}\text { Métodos de investigación, formulación, } \\
\text { análisis y resolución de problemas de } \\
\text { investigación }\end{array}$ \\
\hline $\begin{array}{l}\text { Model of forming future } \\
\text { specialists' research com- } \\
\text { petence }\end{array}$ & Ismuratova et al. (2018) & $\begin{array}{l}\text { Métodos modernos de búsqueda, } \\
\text { procesamiento y uso de la información, } \\
\text { dominar algunos métodos de investigación } \\
\text { científica }\end{array}$ \\
\hline $\begin{array}{l}\text { Developing research com- } \\
\text { petence in undergraduate } \\
\text { students through hands on } \\
\text { learning }\end{array}$ & Davidson y Palermo (2015) & $\begin{array}{l}\text { Diseño de estudio de investigación, desarro- } \\
\text { llo de una revisión sistemática de literatura, } \\
\text { gestión de proyectos de investigación, } \\
\text { comparación de metodologías de investiga- } \\
\text { ción, aplicación de métodos estadísticos de } \\
\text { análisis de datos cualitativos y cuantitativos, } \\
\text { y comunicación de resultados de literatura } \\
\text { científica }\end{array}$ \\
\hline $\begin{array}{l}\text { Formación integral y com- } \\
\text { petencias. Pensamiento } \\
\text { complejo, currículo, didácti- } \\
\text { ca y evaluación }\end{array}$ & Tobón (2013) & $\begin{array}{l}\text { Habilidades lectoras y de escritura, la } \\
\text { gestión de la información, aprendizaje autó- } \\
\text { nomo, espíritu de búsqueda e indagación y } \\
\text { sólidos hábitos de estudio }\end{array}$ \\
\hline $\begin{array}{l}\text { Development of a research } \\
\text { competence in university } \\
\text { students through blended } \\
\text { learning }\end{array}$ & Lagunes et al. (2016) & $\begin{array}{l}\text { Identificar problemas del proyecto de } \\
\text { investigación, análisis cualitativo y cuanti- } \\
\text { tativo de datos, búsqueda de información } \\
\text { relevante, analizar y resolver problemas, } \\
\text { analizar, buscar y evaluar información }\end{array}$ \\
\hline $\begin{array}{l}\text { Las nueve competencias } \\
\text { de un investigador / The } \\
\text { nine powers of an inves- } \\
\text { tigator }\end{array}$ & Rivas (2011) & $\begin{array}{l}\text { Plantear el problema de la investigación, } \\
\text { revisar el estado del arte, conocer técnicas } \\
\text { de análisis de datos, y redactar los resulta- } \\
\text { dos científicos }\end{array}$ \\
\hline
\end{tabular}

Fuente: elaboración propia. 


\section{Administración de literatura bibliográfica}

Una de las actividades fundamentales al hacer una revisión de literatura científica es el registro y la gestión de la información. Esta tarea implica recoger, almacenar, recuperar y administrar toda la información encontrada en las búsquedas bibliográficas. La fundamentación del contenido de un manuscrito con referencias adecuadas juega un papel importante en su aceptación; además, la administración de literatura bibliográfica ayuda a rastrear el origen de un estudio o los métodos adoptados. Por lo tanto, la precisión de las referencias en los manuscritos que se envían a las revistas es un tema relevante que contribuye a la calidad de los documentos bibliográficos (Mueen \& Al Dhubaib, 2011).

\section{Búsqueda de información bibliográfica}

Una búsqueda de información bibliográfica es un proceso que permite encontrar información precisa y eficaz con la intención de obtener los resultados adecuados a las necesidades de información de entre ese gran volumen de documentación existente. Del objetivo de la búsqueda dependerá el tipo de recursos que se deberá consultar, por ejemplo, revistas científicas, actas de congresos, normas, documentales, entre otros (Universitaria, 2013).

\section{Procesamiento y obtención de información}

La obtención de información confiable es un factor valioso para desarrollar trabajos de investigación verídicos y reales. Existen diferentes técnicas e instrumentos orientados a la obtención de información, los cuales abarcan observación, encuestas, entrevistas, listas de chequeo, cuestionarios, entre otros, y todos constituyen un medio para recabar la información necesaria para el objeto de estudio (Leyva, Pérez y Pérez, 2018).

\section{Estadística descriptiva e inferencial}

La estadística descriptiva tiene como objetivo resumir la evidencia encontrada en una investigación de manera sencilla y clara para su interpretación. Se pueden utilizar distintos tipos de recursos como gráficos y tablas que permitan interpretar, señalar tendencias y comparar los datos procesados (Rendón-Macías, Villasís-Keever y Miranda-Novales, 2016). En contraste, el análisis inferencial solo debe usarse en los estudios que comparan los resultados entre dos o más grupos, o bien, que establecen los cambios en un mismo grupo (Flores-Ruiz et al., 2017). Por esto, la estadística, tanto descriptiva como inferencial, se encarga de examinar una muestra de población a fin de recolectar datos y resultados que contribuyan a la obtención de resultados que puedan emplearse en nuevos estudios (Salazar, Castillo y Del Castillo, 2017).

\section{Análisis e investigación de proyectos}

El proceso de análisis e investigación de un proyecto puede darse a conocer de diferentes maneras, como lo refieren Bauer, Bleck-Neuhaus y Dombois (2010), las cuales permiten lograr el enfoque correcto, objetivos definidos con claridad, un diseño de investigación transparente y realista, una forma especial de selección de los métodos científicos, así como un plan de trabajo y de utilización de recursos; esto hace posible que, finalmente, se obtenga un producto exitoso.

\section{METODOLOGÍA}

Para cumplir con los objetivos de nuestra investigación seguimos tres etapas: la determinación de las estrategias instruccionales que aplicaremos, el diseño e implementación de un EVA (que servirá como herramienta para fomentar las capacidades de investigación de los estudiantes), y 
la determinación de la mejora de las capacidades investigativas de los estudiantes participantes en el estudio.

\section{Determinación de las estrategias instruccionales}

Las estrategias instruccionales son consideradas como acciones que los docentes deben crear durante el proceso de enseñanza-aprendizaje para que la construcción de conocimiento y el desarrollo de habilidades sean las óptimas para la formación de los estudiantes (Tarango et al., 2015). En nuestro estudio tomamos como base la publicación de Pástor, Arcos y Gómez (2018), quienes describen las teorías de aprendizaje que, de acuerdo con Wu et al. (2012), han sido las que más han influido en la educación actual y, a su vez, las teorías de diseño instruccional que estarían previstas dentro de cada paradigma de aprendizaje. La tabla 2 resume la propuesta de estos autores.

De las catorce teorías de diseño instruccional que mencionan Pástor et al. (2018), consideramos cuatro, las cuales se constituyeron en estrategias que implementamos en la segunda etapa. Las teorías son: instrucción directa, aprendizaje social, aprendizaje por descubrimiento y teoría de la elaboración; las dos primeras corresponden a la teoría conductista; la tercera, a la teoría cognitiva; y la última, a la teoría constructivista, que describimos a continuación. Esta información servirá de base para el diseño e implementación del EVA, que contribuirá a la formación del estudiantado $\mathrm{y}$ al fortalecimiento de las capacidades de investigación.

\section{INSTRUCCIÓN DIRECTA}

Es un modelo de comunicación unidireccional, basado en los modelos de enseñanza clásicos, que considera que el docente aporta al alumno nuevos conocimientos (Rafael y Salguero, 2009). El objetivo principal es que los estudiantes aprendan, comúnmente se refiere a una habilidad en la que se explica cuál es el objetivo y cómo se realiza una actividad determinada (Martín, 2007). Para que este modelo funcione de la mejor manera, los estudiantes deberán comprometerse de forma directa en cada una de las áreas mediante la formulación de preguntas y una retroalimentación del docente (Sanhueza, 2012).

Tabla 2. Teorías de aprendizaje y diseño instruccional

\begin{tabular}{|c|l|}
\hline TEORÍA DE APRENDIZAJE & \multicolumn{1}{|c|}{ TEORíAS DE DISEÑO INSTRUCCIONAL } \\
\hline \multirow{3}{*}{ Conductivista } & Instrucción directa \\
& Instrucción programada \\
& Aprendizaje social \\
\hline \multirow{2}{*}{ Cognitiva } & Teoría de la atribución \\
& Teoría de la elaboración \\
& Teoría del desarrollo cognitivo \\
& Teoría del desarrollo acondicionado \\
\hline \multirow{3}{*}{ Constructivista } & Aprendizaje basado en problemas \\
& Aprendizaje cognitivo \\
& Aprendizaje por descubrimiento \\
& Aprendizaje basado en casos \\
& Aprendizaje situado \\
& Teoría de la actividad \\
& Teoría actor-red \\
& \\
&
\end{tabular}

Fuente: Pástor et al. (2018). 


\section{APRENDIZAJE SOCIAL}

Es un modelo de aprendizaje en el que se aprende después de haber visto a un sujeto hacerlo. El observador aprende por diversos medios, por ejemplo, a través de los sentidos (Cherem et al., 2015). En este tipo de aprendizaje se distinguen cuatro procesos: atención, retención, producción y motivación; además, integra las teorías del aprendizaje y la personalidad.

\section{APRENDIZAJE POR DESCUBRIMIENTO}

En este tipo de aprendizaje, el docente ofrece a los estudiantes la posibilidad de aprender por sí solos; así, el estudiante fortalece su propio conocimiento de la mano de la enseñanza tradicional, en la cual el docente es el facilitador del conocimiento. El aprendizaje por descubrimiento contribuye de manera significativa al proceso de aprendizaje en los estudiantes universitarios (Eleizalde et al., 2010).

\section{TEORÍA DE LA ELABORACIÓN}

Esta teoría parte del análisis de las estructuras del conocimiento, así como de los procesos de las teorías del aprendizaje. Muestra modelos de diseños más apropiados para ponerlos en práctica, fomenta la enseñanza de forma sistemática a fin de contribuir a reforzar y resumir apropiadamente todo lo que el estudiante ha aprendido (Acaso, 1998).

\section{Diseño e implementación del entorno virtual de aprendizaje}

Con el propósito de mejorar las capacidades y habilidades investigativas en los estudiantes universitarios, diseñamos un EVA mediante Moodle Ver. 3.52, estructurado en cinco secciones, cada una de las cuales corresponden a una capacidad investigativa a fomentar. Cada sección del aula utiliza las estrategias determinadas en la sección anterior, las cuales son llevadas a la práctica a través de los recursos y las actividades disponibles en Moodle.

En la figura de la siguiente página presentamos la estructura de dos secciones del EVA a manera de ejemplo. El apartado a) de la figura corresponde a la competencia 1: administración de literatura bibliográfica, en la que se aplica la estrategia instruccional "instrucción directa" a través de un patrón didáctico para la presentación del tema; se detallan los objetivos de la materia y, en seguida, se muestra un video que trata el tema con aspectos puntuales (objetivo, aplicación), herramientas y actividades de evaluación aplicadas a la práctica.

El apartado b) de la figura corresponde a la competencia 2: procesamiento y obtención de información, en el que aplica la estrategia instruccional "teoría de la elaboración”, mediante la presentación de diferentes componentes: páginas web, recursos tipo libro o wikis, foros de discusión, tareas asignadas y pruebas de evaluación. Una estructura similar se utilizó en las secciones restantes del EVA, de acuerdo con lo especificado en la tabla 3.

\section{Evaluación de las capacidades de investigación}

Los datos para la evaluación de la mejora de las capacidades de investigación fueron recopilados mediante el desarrollo del curso virtual, en el cual se matricularon 65 estudiantes de la carrera de Ingeniería en Sistemas de una universidad ecuatoriana, durante el período septiembre 2018-febrero 2019. Los estudiantes seleccionados cursaban el quinto, sexto y octavo semestre de su carrera respectivamente, en las asignaturas de Aplicaciones web, Ingeniería de software y Diseño de tesis.

Los estudiantes matriculados en el curso virtual estuvieron sometidos al siguiente proceso: evaluación de las capacidades de investigación antes de iniciar el curso (pretest), capacitación de los estudiantes a través del aula virtual y cumplimiento de 

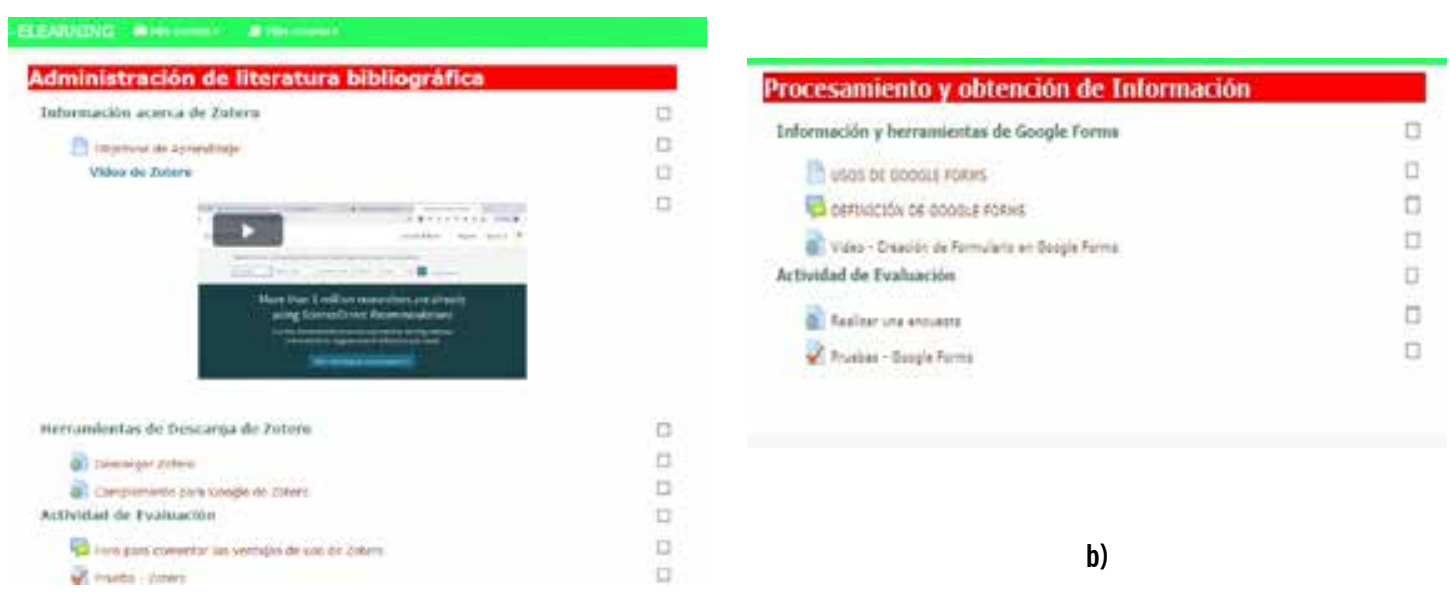

a)

b)

Figura. Estructura del aula virtual y aplicación de las estrategias de diseño instruccional.

Fuente: elaboración propia.

Tabla 3. Aplicación de las estrategias instruccionales para fomentar las capacidades de investigación

\begin{tabular}{|c|c|c|}
\hline CaPACIDAD dE INVESTigación REPRESENTADA en El EVA & ESTRATEGIA INSTRUCCIONAL & RECURSOS/ACTIVIDADES \\
\hline Administración de literatura bibliográfica & Instrucción directa & $\begin{array}{l}\text { - } \text { Video } \\
\text { - Foro de discusión } \\
\text { - Prueba }\end{array}$ \\
\hline Procesamiento y obtención de información & Teoría de la elaboración & $\begin{array}{l}\text { - } \text { Página web } \\
\text { - Foro de discusión } \\
\text { - Videos } \\
\text { - Tareas asignadas }\end{array}$ \\
\hline Búsqueda de información bibliográfica & Aprendizaje por descubrimiento & $\begin{array}{l}\text { - Página web } \\
\text { - Foro de discusión } \\
\text { - Videos } \\
\text { - Tareas asignadas } \\
\text { - Wikis }\end{array}$ \\
\hline Estadística descriptiva e inferencial & Teoría de la elaboración & $\begin{array}{ll}\text { - } & \text { Página web } \\
\text { - } & \text { Recurso tipo libro } \\
\text { - } & \text { Video } \\
\text { - } & \text { Tarea } \\
\end{array}$ \\
\hline Análisis e investigación de proyectos & Aprendizaje social & $\begin{array}{l}\text { - Página web } \\
\text { - Documento didáctico } \\
\text { - Foro de discusión } \\
\text { - Actividad tipo taller }\end{array}$ \\
\hline
\end{tabular}

Fuente: elaboración propia. 
todas las actividades establecidas en el curso, y evaluación de las capacidades de investigación luego de haber cumplido la totalidad de la capacitación (postest). Este proceso fue culminado por 54 estudiantes, es decir, $83.1 \%$ de los matriculados ejecutaron la totalidad de las actividades planteadas en este proceso y once fueron descartados del proceso de evaluación (ver tabla 4).

El objetivo del test de evaluación fue determinar el nivel de habilidad en el desarrollo de las capacidades de investigación mediante preguntas relacionadas con su carrera. El test se conformó por 50 preguntas estructuradas de la siguiente manera: siete para evaluar la competencia de administración de la literatura bibliográfica, 18 para evaluar la competencia de estadística descriptiva e inferencial, ocho para evaluar la competencia de procesamiento y obtención de información, once para evaluar la competencia de análisis e investigación de proyectos, y seis para evaluar la competencia de búsqueda y gestión de la información. Cada pregunta correcta fue evaluada sobre dos puntos, es decir, el test completo tuvo una evalua- ción de 100 puntos; cada competencia evaluada estuvo ponderada como se indica en la tabla 5 .

Una vez recopilados los resultados del pretest y del postest, aplicamos la prueba de rangos con signo de Wilcoxon; esta prueba no paramétrica permite comparar el rango medio de las dos muestras relacionadas y determinar si existen diferencias entre estas. También, evaluamos estadísticamente la diferencia en las variables de interés entre estos grupos mediante las pruebas de Mann Whitney-U. La significancia considerada fue de $\mathrm{P}<0.05$. Este análisis fue realizado para cada grupo y para la totalidad de estudiantes, como ya mencionamos; los estudiantes que completaron las tres etapas del proceso de evaluación fueron incluidos en el análisis y en la estadística descriptiva reportada para cada competencia evaluada.

\section{RESULTADOS}

Un total de 65 estudiantes se matricularon en el curso virtual, de los cuales once no completaron

Tabla 4. Participantes en el curso virtual

\begin{tabular}{|l|c|c|c|}
\hline \multicolumn{1}{|c|}{ AsIGNATURA } & Núm. DE ESTUdIANTES MATRICULAdOS & Núm. DE ESTUDIANTES ELIMINAdOS & TOTAL \\
\hline Aplicaciones web & 37 & 6 & 31 \\
\hline Ingeniería de software & 15 & 3 & 12 \\
\hline Diseño de tesis & 13 & 2 & 11 \\
\hline Total & 65 & 11 & 54 \\
\hline
\end{tabular}

Fuente: elaboración propia.

Tabla 5. Número de preguntas del pre- y postest y su ponderación

\begin{tabular}{|l|c|c|}
\hline \multicolumn{1}{|c|}{ CAPACIdad de Investigación } & Número de PREguntas & PONDERACIÓN (\%) \\
\hline C1. Administración de literatura bibliográfica & 7 & 14 \\
\hline C2. Procesamiento y obtención de información & 18 & 36 \\
\hline C3. Búsqueda de información bibliográfica & 8 & 16 \\
\hline C4. Estadística descriptiva e inferencial & 11 & 22 \\
\hline C5. Análisis e investigación de proyectos & 6 & 12 \\
\hline Total & 50 & 100 \\
\hline
\end{tabular}

Fuente: elaboración propia. 
las actividades planificadas en el pretest, en el curso o en el postest; en cambio, 54 estudiantes consumaron todas las etapas que nos permitían evaluar la mejora en el desarrollo de las capacidades de investigación.

La tabla 6 muestra el análisis de los resultados en el pretest y el postest, correspondientes a los estudiantes de la asignatura Aplicaciones web $(n=31)$. Observamos que existe una diferencia significativa entre los resultados de los dos test; en ambos casos, calculamos el rango intercualtil (IRQ) referente a cada capacidad evaluada, es decir, la diferencia de la mediana entre el primer y el tercer cuartil de los resultados obtenidos, que representamos de la siguiente manera:

$$
\mathrm{M}(\mathrm{Q} 1, \mathrm{Q} 3)
$$

donde:

$\mathrm{M}=$ mediana correspondiente a la capacidad analizada

Q1 = mediana del primer cuartil

Q3 = mediana del tercer cuartil

$$
\mathrm{IRQ}=\mathrm{Q} 3-\mathrm{Q} 1
$$

Por ejemplo, en la capacidad administración de literatura, esta tiene un IRQ pretest 8.34 (7.6, $8.9)=1.3$ y postest de $11.67(10.56,15.50)=2.94$, lo que significa que, en el primer caso, el 50\% intermedio de los datos es 1.3, y en el segundo, 2.94, con un $\mathrm{P}=0.00$, que constituye una diferencia significativa en esta capacidad.

De acuerdo con los resultados expuestos en la tabla 7, todos los grupos de estudio tienen mejoras significativas en las cinco capacidades analizadas, ya que $\mathrm{P}<0.05$.

En el grupo de estudio 1 se evidencia que la capacidad mejor evaluada es $\mathrm{C} 1$ administración de literatura bibliográfica, con una mejora de 3.93 puntos y la evaluación más baja corresponde a $\mathrm{C}_{5}$ análisis e investigación de proyectos, con una mejora de 1.43 puntos. En el grupo 2 se muestra que la capacidad mejor evaluada es $\mathrm{C}_{4}$ estadística descriptiva e inferencial, con una mejora de 3.62, y la evaluación más baja es para C5 análisis e investigación de proyectos, con una mejora de 0.99 puntos. En el grupo 3 advertimos que la capacidad mejor evaluada es $\mathrm{C}_{4}$ estadística descriptiva e inferencial, con una mejora de 4.08 puntos, y la evaluación más baja pertenece a $\mathrm{C}_{3}$ búsqueda y gestión de información, con una mejora de 0.88 puntos.

La baja mejora en la capacidad $\mathrm{C}_{5}$ en los dos primeros grupos puede ser el reflejo de la escasa formación académica de los estudiantes en el tema de desarrollo y gestión de proyectos, que es parte de las asignaturas de séptimo y noveno semestre. La baja mejora en la capacidad $\mathrm{C}_{3}$ correspondiente al grupo 3 era de esperarse, ya que esta capacidad fue bien evaluada en el pretest y el rango de mejora está cerca del límite máximo en el postest.

La tabla 8 y la gráfica contienen los resultados del procesamiento del cálculo de las medias de los 54 estudiantes que formaron parte del estudio. Se concluye que la capacidad mejor evaluada es C4 estadística descriptiva e inferencial, con una mejora de 3.87 puntos, y la evaluación más baja corresponde a $\mathrm{C}_{5}$ análisis e investigación de proyectos, con una mejora de 1.33 puntos.

Los resultados evidencian que las actividades desarrolladas por los estudiantes en el EVA contribuyeron a fortalecer todas las capacidades de investigación. En la tabla 8 podemos apreciar que las medias del postest de estas capacidades están cerca del valor máximo establecido en la ponderación. Advertimos, además, la utilidad del conocimiento proporcionado en el EVA en lo concerniente a la estadística descriptiva e inferencial, impartida en el cuarto semestre de su carrera y que tiende a ser olvidada en los semestres subsiguientes.

\section{CONCLUSIONES}

En nuestro estudio hemos analizado cinco capacidades de investigación que son fundamentales para el proceso de aprendizaje y que deben ser desarrolladas por los estudiantes de pregrado: 


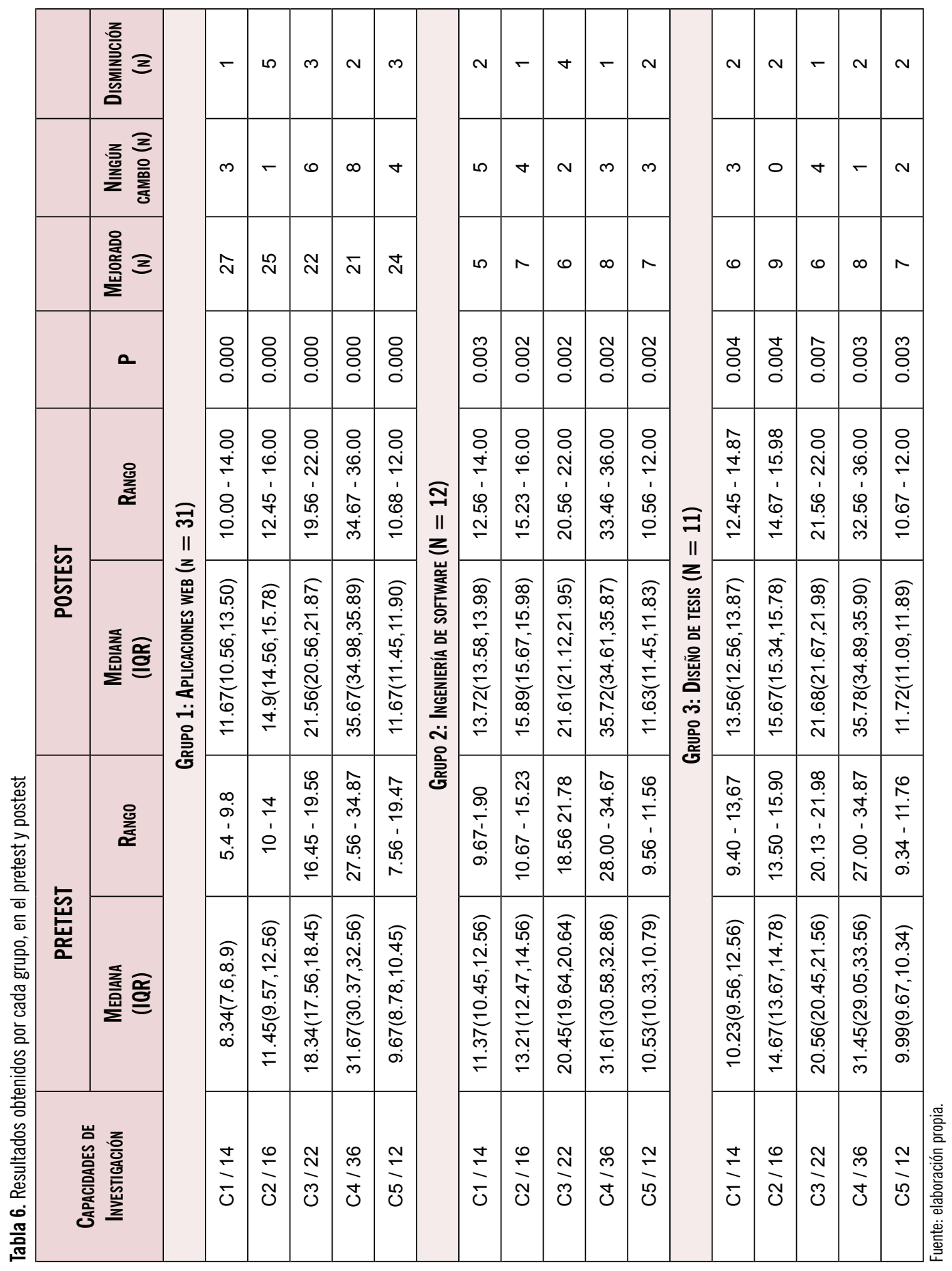


Tabla 7. Resumen de resultados por cada competencia investigativa y grupo estudiado

\begin{tabular}{|c|c|c|c|c|}
\hline \multirow{3}{*}{ TABLA DE RESULTADOS FINALES } & \multirow{3}{*}{$\begin{array}{c}\text { PONDERACIÓN } \\
/ 100\end{array}$} & \multicolumn{3}{|c|}{$N=54$ ESTUDIANTES } \\
\hline & & A PRETEST & B POSTEST & \multirow{2}{*}{ A-B } \\
\hline & & Media & MEDIA & \\
\hline \multicolumn{5}{|c|}{ Grupo 1: ApLICACIONES WEB $(\mathrm{N}=31)$} \\
\hline Administración de literatura bibliográfica & 14 & 8.02 & 11.95 & 3.93 \\
\hline Procesamiento y obtención de información & 16 & 11.38 & 14.93 & 3.55 \\
\hline Búsqueda y gestión de información & 22 & 18.18 & 21.30 & 3.12 \\
\hline Estadística descriptiva e inferencial & 36 & 31.60 & 35.50 & 3.9 \\
\hline Análisis e investigación de proyectos & 12 & 10.20 & 11.63 & 1.43 \\
\hline \multicolumn{5}{|c|}{ Grupo 2: Ingeniería de SOFTWARE (N = 12) } \\
\hline Administración de literatura bibliográfica & 14 & 11.49 & 13.68 & 2.19 \\
\hline Procesamiento y obtención de información & 16 & 13.30 & 15.78 & 2.48 \\
\hline Búsqueda y gestión de información & 22 & 20.31 & 21.51 & 1.2 \\
\hline Estadística descriptiva e inferencial & 36 & 31.63 & 35.25 & 3.62 \\
\hline Análisis e investigación de proyectos & 12 & 10.56 & 11.55 & 0.99 \\
\hline \multicolumn{5}{|c|}{ Grupo 3: Diseño de tesis $(\mathrm{N}=11)$} \\
\hline Administración de literatura bibliográfica & 14 & 10.81 & 13.30 & 2.49 \\
\hline Procesamiento y obtención de información & 16 & 14.50 & 15.56 & 1.06 \\
\hline Búsqueda y gestión de información & 22 & 20.91 & 21.79 & 0.88 \\
\hline Estadística descriptiva e inferencial & 36 & 31.12 & 35.20 & 4.08 \\
\hline Análisis e investigación de proyectos & 12 & 10.12 & 11.53 & 1.41 \\
\hline
\end{tabular}

Fuente: elaboración propia.

Tabla 8. Resumen de resultados por cada capacidad de investigación

\begin{tabular}{|c|c|c|c|c|c|}
\hline \multirow{3}{*}{ Capacidades de INVESTIGACIÓN } & \multirow{3}{*}{$\begin{array}{c}\text { PONDERACIÓN } \\
/ 100\end{array}$} & \multicolumn{4}{|c|}{$\mathrm{N}=54$ ESTUDIANTES } \\
\hline & & A PRETEST & B POSTEST & & \\
\hline & & MeDIA & MEDIA & A-B & $\mathbf{P}$ \\
\hline C1. Administración de literatura bibliográfica & 14 & 9.36 & 12.61 & 3.25 & 0.000 \\
\hline C2. Procesamiento y obtención de información & 16 & 12.44 & 15.25 & 2.81 & 0.000 \\
\hline C3. Búsqueda y gestión de información & 22 & 19.21 & 21.44 & 2.23 & 0.000 \\
\hline C4. Estadística descriptiva e inferencial & 36 & 31.51 & 35.38 & 3.87 & 0.000 \\
\hline C5. Análisis e investigación de proyectos & 12 & 10.26 & 11.59 & 1.33 & 0.000 \\
\hline Total & 100 & 82.78 & 96.27 & 13.49 & \\
\hline
\end{tabular}

Fuente: elaboración propia. 


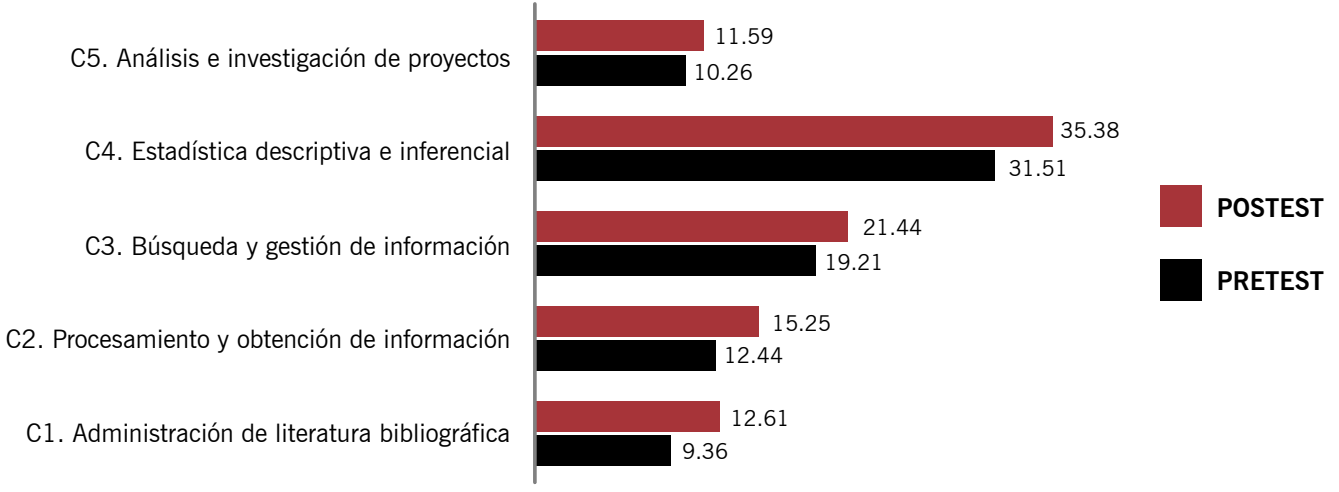

Gráfica. Comparación de los resultados finales del pretest y postest Fuente: elaboración propia.

administración de la literatura bibliográfica, búsqueda de información bibliográfica, proceso y obtención de información, estadística descriptiva e inferencial, y análisis e investigación de proyectos. Para fomentar una mejora de estas, estructuramos un curso virtual con base en cuatro estrategias instruccionales: instrucción directa, aprendizaje social, aprendizaje por descubrimiento y teoría de la elaboración, las cuales fueron implementadas mediante actividades y recursos disponibles en Moodle.

Un total de 54 estudiantes fueron capacitados en el curso virtual y cumplieron con todo el proceso planificado. Los resultados demuestran que todas las capacidades de investigación mejoran significativamente al instruir a los estudiantes en aulas virtuales que utilizan las estrategias instruccionales seleccionadas. Existe una mejora global en la aplicación de las capacidades de investigación del $13.49 \%$, entre estas, la $\mathrm{C}_{4}$ estadística descriptiva e inferencial representa la de mayor porcentaje de mejora.

El propósito de desarrollar capacidades de investigación debe ser impulsado mediante estrategias instruccionales que se acoplen a la forma de enseñanza de la investigación mediante el uso del EVA, de tal forma que esas capacidades logren ser parte integral de los estudiantes universitarios y puedan, posteriormente, aplicarse en estudios de posgrado y contribuyan a solucionar problemas de la sociedad y su entorno.

Podríamos extender las cinco capacidades de investigación propuestas en este trabajo, si consideramos que su ámbito de aplicación son los estudiantes de pregrado y su enseñanza fue de manera virtual; sin embargo, hay que considerar que, para el nivel de posgrado, debemos examinar otros aspectos y características de investigación orientados a la formación de capacidades de investigación científica. Además, podríamos analizar estrategias instruccionales complementarias a las empleadas en nuestro estudio, lo cual abre una perspectiva para representar una misma capacidad o habilidad de investigación con distintas técnicas.

\section{REFERENCIAS BIBLIOGRÁFICAS}

Acaso López Bosch, M. (1998). La teoría de la elaboración como estrategia organizativa dentro del marco de la educación artística como disciplina. Arte, Individuo y Sociedad, 10, 63-76. https:// doi.org/10.5209/ARIS.6807

Miyahira Arakaki, J. M. (2009). La investigación formativa y la formación para la investigación en el pregrado. Revista Médica Herediana, 20(3), 119-122.

Barth, M.; Godemann, J.; Rieckmann, M. \& Stoltenberg, U. (2007). Developing key competencies for sustainable development 
in higher education. International Journal of Sustainability in Higher Education, 8(4), 416-430. https://doi. org/10.1108/14676370710823582

Bauer, Waldemar; Bleck-Neuhaus, Jörn \& Dombois, Rainer. (2010). Desarrollo de proyectos de investigación (DAAD). Recuperado de: https://www.portal.uni-koeln.de/sites/international/ aaa/92/92pdf/92pdf_PROGRANT_Desarrollo_de_proyectos de_investigacion_SCREEN.pdf

Beckerleg, M. \& Collins, J. (2007). Producing research from undergraduate projects. Trabajo presentado en la AaeE Conference. Melbourne.

Cherem, A.; Claudia, H.; Reyna, G.; García, D.; Morales, A.; Denisse Gómez, A.; Dalia, R.; Estrada, G.; Ruiz, D.; Salgado Martinez, A.; Sánchez, E.; Cintia, 0. y García Mendoza, S. (2015). Aprendizaje social de Albert Bandura: marco teórico. México: Facultad de Estudios Superiores Acatlán, Universidad Nacional Autónoma de México.

Coates, H. (2014). Higher education learning outcomes assessment. Peter Lang. Recuperado de: https://www.peterlang. com/view/title/17378

Davidson, Z. E. \& Palermo, C. (2015). Developing research competence in undergraduate students through hands on learning [Research article]. Journal of Biomedical Education. https:// doi.org/10.1155/2015/306380

Eleizalde, M.; Parra, N.; Palomino, C.; Reyna-Bello, A. y Trujillo, I. (2010). Aprendizaje por descubrimiento y su eficacia en la enseñanza de la biotecnología. Revista de Investigación, 34, 271-290.

Emelyanova, I.; Teplyakova, 0. \& Boltunova, L. (2017). The students' research competences formation on the Master's Programmes in Pedagogy. European Journal of Contemporary Education, 6(4), 700-714.

Flores-Ruiz, E.; Miranda-Novales, M. G.; Villasís-Keever, M. Á.; Flores-Ruiz, E.; Miranda-Novales, M. G. y Villasís-Keever, M. Á. (2017). El protocolo de investigación VI: Cómo elegir la prueba estadística adecuada. Estadística inferencial. Revista Alergia México, 64(3), 364-370. https://doi.org/10.29262/ram. v64i3.304

García-Gutiérrez, Z. del P. y Aznar-Díaz, I. (2018). El desarrollo de competencias investigativas, una alternativa para formar profesionales en pedagogía infantil como personal docente investigador. Revista Electrónica Educare, 23(1). https://doi. org/10.15359/ree.23-1.15

González González, J. (2004). Los paradigmas de la calidad educativa: de la autoevaluación a la acreditación. México: UDUAL.
Hughes, G. (2019). Developing student research capability for a 'post-truth' world: Three challenges for integrating research across taught programmes. Teaching in Higher Education, 24(3), 394-411. https://doi.org/10.1080/13562517.2018.15 41173

Ismuratova, S. I.; Slambekova, T. S.; Kazhimova, K. R.; Alimbekova, A. A. \& Karimova, R. E. (2018). Model of forming future specialists' research competence. Revista Espacios, 39(35). Recuperado de: http://www.revistaespacios.com/ a18v39n35/18393524.html

Lagunes, A.; Judikis, J.C. \& Flores, M. A. (2016). Development of a research competence in university students through blended learning. TOJET: The Turkish Online Journal of Educational Technology, INTE, 668-673.

Lau, J.; Cortes, J.; Marzal, M.; Tarango, J. y Pirela, J. (2015). Demanda y competencias informativas de academicos (DECIA). Una caracterizacion internacional. Proyecto de investigacion. CETYS. Recuperado de: https://docs.google.com/uc?authuser =0\&id = OB1 5E8GlqcJJMG9KRkMtRzQ1c2c\&export=download

Leyva López, H. P.; Pérez Vera, M. G. y Pérez Vera, S. M. (2018). Google Forms en la evaluación diagnóstica como apoyo en las actividades docentes. Caso con estudiantes de la Licenciatura en Turismo. RIDE. Revista Iberoamericana para la Investigación y el Desarrollo Educativo, 9(17), 84-111. https://doi. org/10.23913/ride.v9i17.374

Martín Recio, Francisco Jesús. (2007). ilnstrucción directa 0 indagación en educación física? Innovación y experiencias educativas.

Moore, J.; Crozier, K. \& Kite, K. (2012). An action research approach for developing research and innovation in nursing and midwifery practice: Building research capacity in one NHS foundation trust. Nurse Education Today, 32(1), 39-45. https://doi. org/10.1016/j.nedt.2011.01.014

Mueen Ahmed, K. \& Al Dhubaib, B. (2011). Zotero: A bibliographic assistant to researcher. Journal of Pharmacology and Pharmacotherapeutics, 2(4), 303. https://doi.org/10.4103/0976500X.85940

Pástor, D.; Arcos, G. y Gómez, 0. (2018). Construcción de plantillas pedagógicas en un curso en línea mediante la aplicación de teorías de diseño instruccional. En Aplicaciones de la tecnología en los procesos educativos. Recuperado de: https:// www.transformacion-educativa.com/attachments/article/256/ Aplicaciones\%20de\%20la\%20tecnolog\%C3\%ADa\%20en\%20 los\%20procesos\%20educativos.pdf 
Rafael, A. y Salguero, C. (2009). La instrucción directa o reproducción de modelos como metodología de enseñanza en el área de educación física. Revista Digital de Educación Física, 1(1).

Rendón-Macías, M. E.; Villasís-Keever, M. Á. y Miranda-Novales, M. G. (2016). Descriptive statistics/Estadística descriptiva. Revista Alergia Mexico, 63, 397-407.

Reyes, 0. (2013). Desarrollo de habilidades investigativas de los estudiantes que cursan el bachillerato en línea. Revista Mexicana de Bachillerato a Distancia, 5(10). http://dx.doi.org/10.22201/ cuaed.20074751e.2013.10.44233

Rivas Tovar, L. A. (2011). Las nueve competencias de un investigador. Investigación Administrativa, 108.

Salazar Pinto, C.; Castillo Galarza, S. del y Del Castillo Galarza, S. (2017). Fundamentos básicos de estadística. Recuperado de: http://www.dspace.uce.edu.ec/handle/25000/13720

Sanhueza, 0. (2012). El modelo de enseñanza directa, el aula taller y algunos problemas de la didáctica. Recuperado de: Catálogo Digital de Publicaciones DC. https://fido.palermo.edu/ servicios_dyc/publicacionesdc/vista/detalle_articulo.php?id_ libro $=443 \&$ id_articulo $=9147$

Tarango, J.; Tirado, A.; Pirela, J. y Osuna, C. (2015). Demanda y competencias informativas de académicos (DECIA). Una caracterización internacional. Proyecto de investigación. Universidad Veracruzana. Coordinado por el doctor Jesús Lau. Instituto de Ingeniería - Región Veracruz/Dirección General de Desar.

Tobón, D. S. (2013). Formación integral y competencias. Pensamiento complejo, currículo, didáctica y evaluación. ECOE.

Trostle, J. (1992). Research capacity building in international health: Definitions, evaluations and strategies for success. Social Science \& Medicine, 35(11), 1321-1324. https://doi. org/10.1016/0277-9536(92)90035-0

Universitaria, B. (2013). La búsqueda de información científica. Recuperado de: http://rua.ua.es/dspace/handle/10045/33983

Wu, W.-H.; Chiou, W.-B.; Kao, H.-Y.; Alex Hu, C.-H. \& Huang, S.-H. (2012). Re-exploring game-assisted learning research: The perspective of learning theoretical bases. Computers \& Education, 59(4), 11531161. https://doi.org/10.1016/j.compedu.2012.05.003

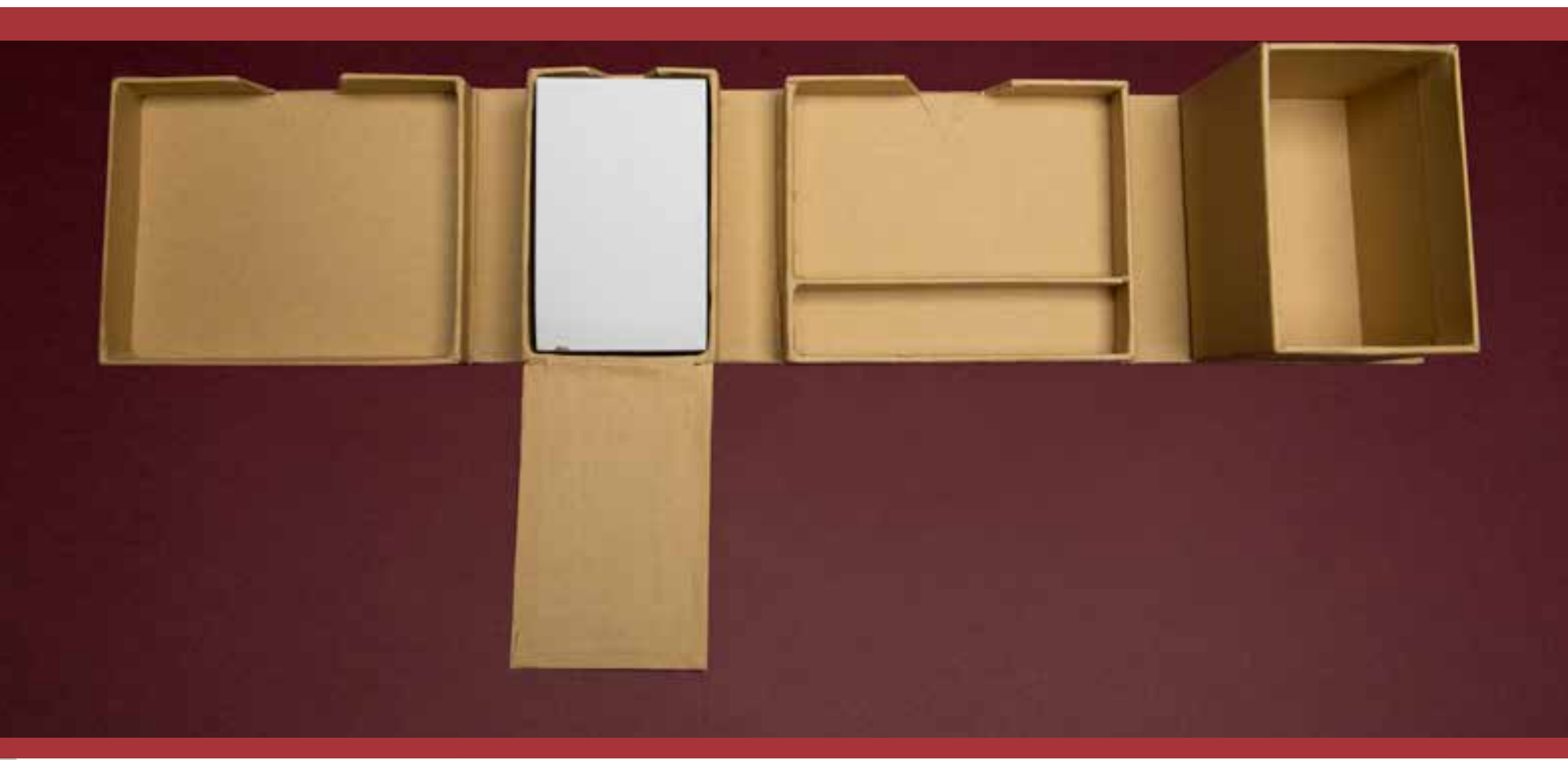

Este artículo es de acceso abierto. Los usuarios pueden leer, descargar, distribuir, imprimir y enlazar al texto completo, siempre y cuando sea sin fines de lucro y se cite la fuente.

\section{CÓMO CITAR ESTE ARTíCULO}

Pástor Ramírez, Danilo; Arcos Medina, Gloria de Lourdes y Lagunes Domínguez, Agustín. (2020). Desarrollo de capacidades de investigación para estudiantes universitarios mediante el uso de estrategias instruccionales en entornos virtuales de aprendizaje. Apertura, 12(1), pp. 6-21. http:// dx.doi.org/10.32870/Ap.v12n1.1842 